RICYDE. Revista Internacional de Ciencias del Deporte doi: $10.5232 /$ ricyde

Rev. int. cienc. deporte

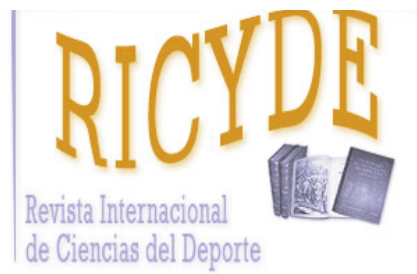

RICYDE. Revista Internacional de Ciencias del Deporte VOLUMEN XIII - AÑO XIII

Paginas:409-425 ISSN:1885-3137

Número 50 - Octubre - 2017

\title{
Propuesta Metodológica del Entrenamiento de la Resistencia en Baloncesto mediante la Modificación de Factores Formales y Estructurales del Juego \\ Methodological Proposals for Endurance Training in Basketball by Modifying Structural and Formal Aspects of the Game
}

\author{
Arnau Sacot ${ }^{1,2}$, Jesús Escosa ${ }^{1,2}$, Alexander T. Latinjak ${ }^{1,2,3}$
}

\author{
1.Escuela Universitaria de la Salud y del Deporte (EUSES), Universitat de Girona. España \\ 2. Escola de Basquet Girona Marc Gasol. España \\ 3.University of Suffolk. United Kingdom
}

\section{Resumen}

El objetivo del presente artículo fue plantear una propuesta metodológica para equipos técnicos en relación a la utilización y modificación de los diferentes parámetros del juego para el trabajo de la resistencia específica en baloncesto desde una perspectiva integrada. Para ello, se elaboró una revisión en relación al estado actual sobre los juegos en espacio reducido en baloncesto, estudiando el efecto de las distintas variables del juego sobre la frecuencia cardíaca y la intensidad de juego. Durante el proceso de selección de artículos de la revisión, se consultaron diversas palabras claves como conditioning y basketball en bases de datos como Pubmed e ISI web of knowledge. Finalmente, se incluyeron en la revisión 12 artículos que evidenciaron que la modificación de parámetros de juego como el número de jugadores, el espacio, el tiempo y las normas, pueden condicionar la orientación del trabajo de la resistencia. En base a estas conclusiones, se formuló una propuesta metodológica sobre cómo utilizar los distintos parámetros de juego con el fin de planificar y/o controlar las cargas de resistencia específica en baloncesto de forma integrada en el juego. Sin embargo, se debe tener presente que los estudios en los que se ha fundamentado la propuesta, únicamente han analizado efectos simples de los diferentes parámetros. De este modo, futuros estudios deberían estudiar los efectos de interacción entre los diferentes parámetros para validar la propuesta. Aun así, creemos que ésta propuesta puede ayudar a integrar el entrenamiento de la resistencia con la técnica y la táctica.

Palabras clave: Deportes de equipo; entrenamiento mediante el juego; entrenamiento de la resistencia; metodología integrada.

\begin{abstract}
The aim of the present study was to create a methodological proposal for coaching staff, which consist in using the game parameters during small sided games in order to integrate endurance training with technical and tactical drills in basketball. For this reason, a systematic review was carried out so as to study the changes in heart rate and game intensity depending on the game parameters used during the game situation. Different key words such as conditioning and basketball in different data bases such as Pub Med or ISI web of knowledge were checked throughout the selection process. Finally, 12 articles were included in the review showing that the modification of some game parameters such as number of players, space conditions, time aspects and game rules, can influence the orientation of the endurance training in basketball. Thereby, a methodological proposal was created to illustrate the usefulness of the different game parameters in order to control and plan the endurance training integrated with technical and tactical drills in basketball. However, the background studies of the methodological proposal should be considered since these studies only have analyzed the simple effects of the different parameters and due to the fact, future studies should study the interaction of the different parameters in order to validate the proposal. Nevertheless, it is believed that this methodological proposal can be helpful so as to integrate endurance training with technical and tactical drills.
\end{abstract}

Key words: Team sports; game-based training; endurance training; integrated methodology.

Correspondencia/correspondence: Arnau Sacot

Escuela Universitaria de la Salud y del Deporte (EUSES), Universitat de Girona. España

Escola de Basquet Girona Marc Gasol. España

Email: arnau.sacot@gmail.com 
Sacot, A.; Escosa, J., y Latinjak, A. T. (2017). Propuesta Metodológica del Entrenamiento de la Resistencia en Baloncesto mediante la Modificación de Factores Formales y Estructurales del Juego. RICYDE. Revista internacional de ciencias del deporte, 50(13), 409-425. https://doi.org/10.5232/ricyde2017.05007

\section{Introducción}

$\mathrm{E}$ baloncesto es un deporte de equipo con una elevada exigencia técnica, táctica y física de forma integrada en múltiples acciones que se desarrollan en un periodo de tiempo muy corto, condicionado por las reglas, los sistemas y estilos de juego (Ben Abdelkrim, Castanga, El Fazaa, y El Ati, 2010b; Klusemann, Pyne, Hopkins, y Drinkwater, 2013). Desde la perspectiva de los sistemas dinámicos, el baloncesto es un deporte de carácter no lineal, donde emergen diferentes acciones técnicas y tácticas a través de la interacción de los diferentes subsistemas del organismo con los oponentes y los compañeros de equipo, hecho que provocará que los contextos donde se desarrollen más todos estos elementos técnicos, tácticos y físicos, sean los más cercanos a la situación real de partido (Balagué, Torrents, Pol, y Seirul·lo, 2014; Davids, Araújo, Correia, y Vilar, 2013).

En este sentido, una de las propuestas metodológicas más interesantes y más utilizadas por los entrenadores y preparadores físicos son los juegos en espacios reducidos (Atli, Köklü, Alemdaroglou, y Koçak, 2013; Castagna, Impellizzeri, Chaouachi, Ben Abdelkrim, y Manzi, 2011; Conte, Favero, Lupo, Francioni, Capranica, y Tessitore., 2016; Delextrat y Kraiem, 2013; Halouani, Chtourou, Gabbett, Chaouachi y, Chamari, 2014; Klusemann y col., 2012; Schelling y Torres-Ronda, 2013). A lo largo de los años, el entrenamiento mediante el juego ha sido muy utilizado como método de trabajo técnico y táctico en el baloncesto, si bien, en la actualidad, estos juegos en espacio reducido han aumentado su popularidad como una tipología de entrenamiento de las capacidades condicionales con una orientación especial y competitiva, concretamente de la resistencia (Atli y col., 2013; Castagna y col., 2011; Conte y col., 2016; Delextrat y Kraiem, 2013; Halouani y col., 2014; Klusemann y col., 2012; Schelling y Torres-Ronda, 2013). Los estudios llevados a cabo por los diferentes autores muestran que la modificación de distintos parámetros relacionados con el juego como el número jugadores implicados, el espacio utilizado para jugar, las diferentes acciones técnicas implicadas, las normas en el juego, la organización del tiempo (intermitente o continuo) y las ratios de trabajo-descanso, provocaron un aumento o una disminución de la frecuencia cardiaca (FC) de los jugadores y un efecto sobre la intensidad del juego (Atli y col., 2013; Conte y col., 2016; Conte y col., 2015b; Delextrat y Kraiem, 2013; Klusemann y col., 2012).

En esta dirección, se encuentran estudios que han analizado algunas situaciones concretas de juego durante el entrenamiento $(1 \times 1,2 \times 2,3 \times 3,4 \times 4,5 \times 5$, superioridades e inferioridades, situaciones defensivas específicas) con la finalidad de observar el comportamiento de ciertas variables fisiológicas entre variables iguales aisladamente (Dehesa, Vaquera, García-Tormo y Bayón, 2015; Torres-Ronda, Ric, Llabres-Torres, de las Heras y Schelling, 2016; Reina, Mancha, Feu y Ibañez, 2017; Vaquera, Suarez, Vidania y Calleja, 2017). Los hallazgos de estos autores sugieren que, aunque se trata de una herramienta muy interesante y próxima a la competición, los juegos en espacio reducido no permiten llegar a cargas iguales o superiores a la situación de competición (Reina y col., 2017). Sin embargo, coinciden en que permiten una transferencia directa a la competición, siendo las situaciones de 3x3 y 4x4 las que ofrecen una mayor aproximación (Torres-Ronda y col., 2016; Vaquera y col., 2017). Además, Dehesa y col. (2015), establecen que la defensa individual promueve mayores valores de FC que la situación de defensa zonal. 
Sacot, A.; Escosa, J., y Latinjak, A. T. (2017). Propuesta Metodológica del Entrenamiento de la Resistencia en Baloncesto mediante la Modificación de Factores Formales y Estructurales del Juego. RICYDE. Revista internacional de ciencias del deporte, 50(13), 409-425. https://doi.org/10.5232/ricyde2017.05007

En este sentido, muchos autores han utilizado la FC como herramienta de control de la carga interna de los juegos en espacio reducido (Castagna y col., 2011; Klusemann y col., 2012; Atli y col., 2013; Delextrat y Kraiem, 2013; Halouani y col., 2014; Dehesa y col., 2015; Conte y col., 2016; Torres-Ronda y col., 2016; Reina y col., 2017; Vaquera y col., 2017). Aun así, sería necesario examinar la validez de la FC como método único de control de la carga interna en baloncesto puesto que se considera que los movimientos en la extremidad superior y los cambios constantes de intensidad que existen durante el juego en baloncesto, promueven una sobreestimación y una alteración de la FC (Nazaraki, Berg, Stergiou, y Chen, 2008; Fox, Scanlan y Stanton, 2017). Por este motivo, Fox y col. (2017), establecen que la FC debería formar parte de las herramientas de control de la carga interna en baloncesto aunque debería ser comparada y contrastada mediante otros métodos de control del entrenamiento.

Con el fin de conseguir un buen ajuste de los parámetros según las necesidades de los jugadores del equipo y del juego, será muy importante conocer las demandas del baloncesto. Desde el punto de vista condicional, se trata de un deporte con elevadas exigencias de esfuerzos de alta intensidad durante todo el partido y sobre todo al final (Klusemann y col., 2013; Schelling y Torres-Ronda, 2013). Aun así, existe una gran variabilidad en las características condicionales específicas en función de la posición que ocupa el jugador en la pista (base, escolta, alero, alero-pívot y pívot), el nivel competitivo, la edad, el tipo de competición (torneo o partido de la temporada) y el género (Nikolaidis, Calleja-González, y Padulo, 2014; Conte y col., 2015a; Delextrat, Biadella, Saavedra, Matthew, Schelling, y Torres-Ronda, 2015; Matthew y Delextrat, 2009; Nazaraki y col., 2008; Klusemann y col., 2013).

Debido a la complejidad que se da por todos los elementos que conforman el juego, existe cierta dificultad en el momento de definir el sistema energético predominante en este deporte (Schelling y Torres-Ronda, 2013). En la actualidad la mayoría de autores (Ben Abdelkrim, Castagna, Jabri, Battikh, El Fazaa, y El Ati, 2010a; Conte y col., 2015b; Delextrat, Baliqi, y Clark, 2013; Delextrat y Martínez, 2014; Delextrat y col., 2015; Klusemann y col., 2013; Schelling y Torres-Ronda, 2013) coinciden en que el baloncesto es un deporte aeroanaeróbico, donde se producen numerosos cambios constantes de intensidad con un componente anaeróbico predominante, como por ejemplo esprints lineales, saltos y cambios de dirección, junto con unas necesidades aeróbicas muy importantes para recorrer las distancias que requiere un partido y recuperarse de los esfuerzos anaeróbicos intermitentes que se producen a lo largo del juego.

A partir de aquí, según los resultados obtenidos por los diferentes autores y en función de los cambios en la FC y la intensidad del juego según los parámetros del juego que se modifican, se puede incidir en la mejora de la resistencia aero-anaeróbica y de las habilidades técnicas específicas del baloncesto (Conte y col., 2016; Delextrat y Martínez, 2014; Schelling y Torres-Ronda, 2013). Además, partiendo de la base de la perspectiva estructuralista, podríamos incidir en diferentes estructuras del organismo mediante los juegos en espacio reducido (Seirul·lo, 1987), puesto que esta concepción entiende el entrenamiento desde una perspectiva holística e integrada basada en el juego, en el cual el organismo, desde un punto de vista hipercomplejo, está formado por diferentes estructuras (condicional, cognitiva, coordinativa, socio-afectiva, emotivo-volitiva y creativo-expresiva) que se interrelacionan entre sí durante las distintas tareas de entrenamiento y en la situaciones de competición. 
Sin embargo, hasta dónde alcanza el conocimiento de los autores, no se ha elaborado ninguna propuesta metodológica sobre el efecto de los diferentes parámetros sobre la resistencia específica en baloncesto en función de la $\mathrm{FC}$ del jugador y/o de la intensidad del juego. Así pues, el objetivo principal del presente artículo fue plantear una propuesta metodológica para equipos técnicos en relación a la utilización y modificación de los diferentes parámetros del juego para el trabajo de la resistencia específica en baloncesto desde una perspectiva integrada. Aun así, para poder establecer una propuesta adecuada, se elaboró una revisión en relación al estado actual sobre los juegos en espacio reducido en baloncesto, estudiando el efecto de las distintas variables del juego sobre variables internas (FC) y externas (intensidad del juego). Por este motivo, en una primera parte del artículo, se analizaron los estudios en relación a los juegos en espacio reducido en baloncesto, explorando la influencia de los distintos parámetros que se pueden modificar sobre la FC y/o la intensidad del juego.

\section{Método}

\section{Muestra}

Para la parte de la revisión se buscaron sistemáticamente artículos sobre parámetros de FC y/o intensidad de juego en juegos en espacio reducido en el baloncesto. Para poder ser incluidos en la revisión, los artículos debían ser de entre 2010 y mayo de 2017. Además, solamente se incluyeron artículos publicados en inglés o castellano y con acceso al texto completo. Los artículos excluidos del análisis fueron los que no estudiaban el baloncesto, los que no se focalizaban en juegos en espacio reducido, y finalmente, se excluyeron los artículos que no analizaban la FC y/o la intensidad durante los juegos en espacios reducidos (Figura 1).

La búsqueda se llevó a cabo en las bases de datos electrónicas PubMed, ISI Web of Knowledge, ERIC, PsycInfo y Dialnet, desde septiembre de 2015 hasta el 15 mayo de 2017. Mediante la búsqueda en las diferentes bases de datos se hallaron un total de 61 artículos de los cuales 12 cumplieron los

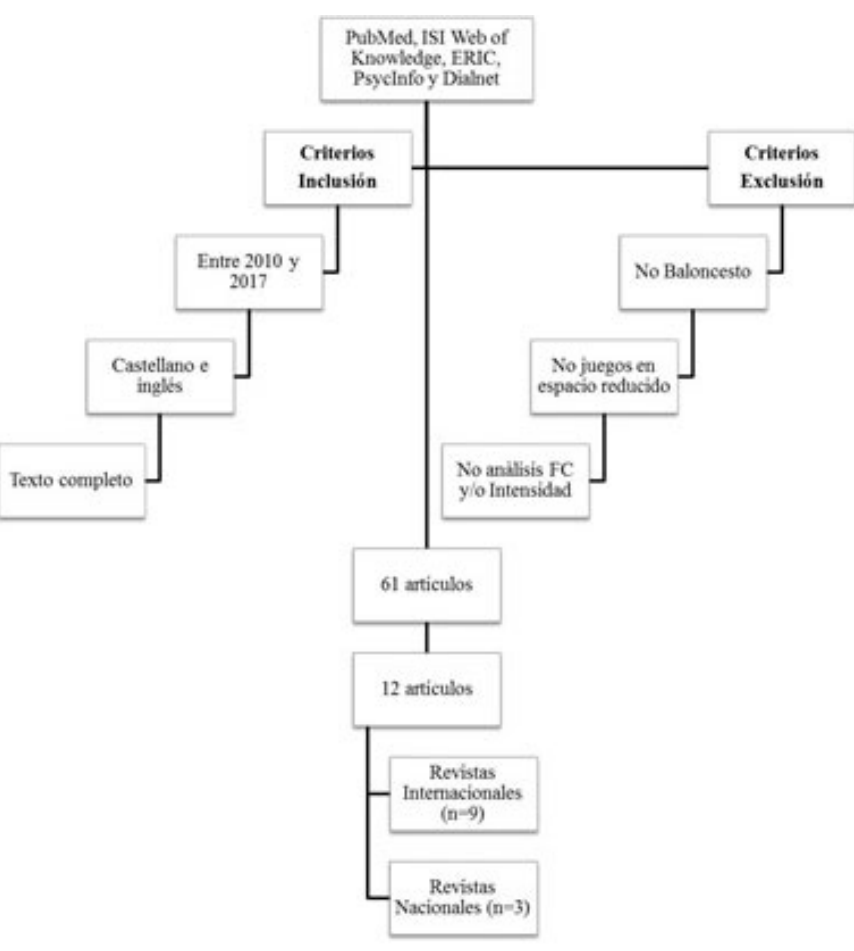

Figura1. Esquema método de búsqueda revisión criterios de inclusión.

Estos artículos fueron publicados entre 2010 y 2017, sobretodo en revistas internacionales $(n=9)$ como el Journal of Strenght and Conditioning Research $(n=4)$ o Journal of Sports Sciences $(n=2)$, pero también en la Revista de Psicología del Deporte $(n=3)$. 
Sacot, A.; Escosa, J., y Latinjak, A. T. (2017). Propuesta Metodológica del Entrenamiento de la Resistencia en Baloncesto mediante la Modificación de Factores Formales y Estructurales del Juego. RICYDE. Revista internacional de ciencias del deporte, 50(13), 409-425. https://doi.org/10.5232/ricyde2017.05007

\section{Procedimientos}

Las principales palabras clave utilizadas para realizar la búsqueda fueron: small-sided games and basketball, conditioning and basketball, skill-based training and basketball, game-based training and basketball, game mode y diferentes combinaciones entre las diferentes palabras clave. En el momento de seleccionar los diferentes artículos que se incluyeron en la revisión, se analizaron los resúmenes de los 61 artículos y en el caso que cumplieran con los criterios de inclusión se procedió a la lectura completa del artículo por parte del primer autor. En el momento que se unieron en el proyecto, el resto de los autores también examinó los artículos seleccionados. Para completar la búsqueda, también se examinó la lista de referencias de los diferentes artículos escogidos. Una vez seleccionados los 12 artículos sometidos a revisión, se procedió a una lectura completa con el fin de conocer la estructura principal de cada uno de los artículos, analizando los parámetros y variables utilizadas en cada uno de los estudios. Después de la primera lectura de todos los artículos, se llevó a cabo una segunda lectura planteando dos preguntas concretas a los estudios realizados. En primer lugar, qué variables independientes habían considerado para estudiar su efecto sobre la FC y/o la intensidad de juego, y en segundo lugar cuál había sido el efecto de las variables independientes sobre la FC y/o la intensidad de juego.

\section{Variables}

A partir de este momento, se extrajeron las variables independientes analizadas en los diferentes estudios y se observó su efecto sobre la FC y/o la intensidad de juego. Posteriormente, se presentó una estructura que agrupaba las variables independientes en parámetros de juego junto con un resumen de su efecto observado sobre la FC y/o la intensidad de juego. Los distintos parámetros de juego analizados por los diferentes estudios fueron utilizados para fundamentar la propuesta metodológica. Partiendo de estos parámetros de juego y una vez observados sus efectos sobre la FC y/o la intensidad de juego, fueron utilizados como base de la propuesta metodológica.

\section{Análisis de datos}

La primera propuesta de la estructura fue elaborada de forma inductiva, por el primer autor de éste trabajo, partiendo de las variables independientes analizadas en los diferentes estudios. Posteriormente, el segundo autor de este trabajo, un experto en entrenamiento condicional en el baloncesto, se leyó en profundidad los artículos para revisar la primera propuesta y discutir modificaciones a la estructura de los parámetros de juego y su efecto sobre la $\mathrm{FC}$ y/o intensidad de juego. Finalmente, el proceso se completa por la revisión de un experto en investigación en ciencias del deporte, que hasta este punto no estuvo involucrado en la elaboración de éste trabajo, que revisó la lógica interna del esquema de parámetros y efectos elaborado anteriormente. 
Sacot, A.; Escosa, J., y Latinjak, A. T. (2017). Propuesta Metodológica del Entrenamiento de la Resistencia en Baloncesto mediante la Modificación de Factores Formales y Estructurales del Juego. RICYDE. Revista internacional de ciencias del deporte, 50(13), 409-425. https://doi.org/10.5232/ricyde2017.05007

\section{Resultados y Discusión}

A lo largo de la revisión emergieron una serie de parámetros de juego a partir de los cuales los autores de los estudios analizados han comparado tanto la FC como la intensidad en situaciones de juego. La mayoría de los estudios se centraron en el número de jugadores, que varía tanto en número como en igualdad o desigualdad numérica entre equipos. Otro parámetro de juego frecuentemente analizado hace referencia al espacio de juego, donde se comparan situaciones a medio campo con situaciones a todo el campo. Finalmente, también se pueden observar parámetros referentes a la modificación de normas y a la organización del tiempo ya sea en tiempo total o el método de organización del tiempo. En los siguientes párrafos se discute el efecto de la variación de los distintos parámetros del juego sobre la FC y la intensidad de juego.

Número de jugadores. Se han estudiado los juegos en espacio reducido en situaciones de 1x1, $2 \times 2,3 \times 3,4 \times 4,5 \times 5$ y en superioridades e inferioridades numéricas (Tabla 1). El análisis de los datos muestra unos valores más elevados FC (e.g., 92.0 $\pm 5.6 \% \mathrm{FCmax} ; 2 \mathrm{2} 2 ; 87.1 \pm 2.9$ \%FCmax; 2x2; Castagna y col., 2011; Conte y col., 2016) y mayor intensidad del juego (e.g., 23.7 \pm 6.1 Total acciones bote; $21.9 \pm 5.4$ Total acciones de pase; $2 \times 2$; Conte y col., 2016) en situaciones con menor número de jugadores. Por otro lado, en las situaciones de juego con

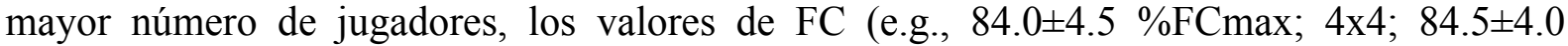
\%FCmax; 4x4; Klusseman y col., 2012; Conte y col., 2016) e intensidad (e.g., 14.4 \pm 7.2 Total acciones bote; $17.7 \pm 6.7$ Total acciones pase; 4x4; Conte y col., 2016) fueron inferiores. De este modo, los resultados obtenidos por los diferentes autores indican un efecto de la variable número de jugadores sobre los valores de FC e intensidad, siendo superiores cuando menor es el número de jugadores (Dehesa y col., 2015; Torres-Ronda y col., 2016; Vaquera y col., 2017). En este sentido, Martínez-Fernández, García e Ibáñez (2015) mostraron más posesiones de juego cuando menor era el número de jugadores, hecho que podría promover esta mayor FC. Aun así, estos autores hallaron un mayor número de pases en las situaciones de $4 \times 4$ en comparación con las situaciones de 3x3, contrariamente a otros estudios analizados.

Los resultados obtenidos en situaciones con superioridades e inferioridades numéricas no permiten la extracción de conclusiones definitivas puesto que los diferentes estudios difieren en cuanto a su efecto sobre la FC. Por un lado, Dehesa y col., (2015) mostraron unos valores elevados de FC (e.g., 91.44 \pm 5.58 \%FCmax; Dehesa y col., 2015), además, Torres-Ronda y col., (2016) hallaron similitudes entre las demandas de FC en competición y las superioridades numéricas. Sin embargo, el estudio de Vaquera y col. (2017) no encontró valores de FC significativamente superiores en comparación con situaciones de igualdad durante la ejecución de un $3 \times 2$ continuado. Aun así, existen pocos estudios en relación a superioridades e inferioridades, hecho que limita las conclusiones respecto a estos tipos de situaciones.

Espacio de Juego. La mayoría de estudios fueron llevados a cabo en situaciones de medio campo (15x14m) y todo el campo (28x15m; Atli y col., 2013; Castagna y col., 2011; Conte y col., 2016; Conte y col., 2015; Dehesa y col., 2015; Klusseman y col., 2012; Montgomery y col., 2010), aunque hay algunos estudios (Delextrat y Martinez, 2014; Delextrat y Kraiem, 2013) que también han dividido el campo en dos partes en vertical (28x7.5m; Tabla 1). No obstante, solo se dispone de dos estudios que contrastaron de forma directa la FC y la intensidad del juego en situaciones de oposición en medio y todo el campo (Atli y col., 2013; Klusseman y col., 2012). Los resultados fueron inconsistentes en cuanto a la diferencia de FC en función del tamaño del campo utilizado. Atli y col. (2013) encontraron diferencias significativas que revelaron una relación entre la variable espacio y la $\mathrm{FC}$, encontrando que a 
Sacot, A.; Escosa, J., y Latinjak, A. T. (2017). Propuesta Metodológica del Entrenamiento de la Resistencia en Baloncesto mediante la Modificación de Factores Formales y Estructurales del Juego. RICYDE. Revista internacional de ciencias del deporte, 50(13), 409-425. https://doi.org/10.5232/ricyde2017.05007

mayor espacio, mayor era la FC (e.g., 85.6 \pm 3.1 \%FCmax; Todo el campo; 76.3 \pm 2.5 \%FCmax; Medio campo; 3x3; Atli y col., 2013), mientras que, Klusseman y col. (2012), no encontraron diferencias significativas en cuanto a la FC. Respecto a la intensidad de juego ambos estudios coinciden en que, a menor espacio, mayor es la intensidad del juego (e.g., 16.8 \pm 3.1 Total acciones de pase; $14 \pm 6.5$ Total acciones de pase; Medio campo; 3x3; 4x4; Atli y col., 2013; Klusseman y col., 2012), contrariamente, a mayor espacio, menor es la intensidad del juego (e.g., 11.3 \pm 2.5 Total acciones de pase; 12.5 \pm 5.5 Total acciones de pase; Todo el campo; 3x3; 4x4; Atli y col., 2013; Klusseman y col., 2012). A falta de más estudios que contrasten diferentes medidas de campo, se han comparado los diferentes estudios llevados a cabo en situaciones a todo el campo y los estudios en situaciones a medio campo con el mismo número de jugadores. Se puede observar que cuando se utilizan espacios más grandes, mayor es la FC del jugador (e.g., 88.0土8.4; \%FCmax; $3 \times 3$ toda pista; Castagna y col., 2011). Por lo contrario, en las situaciones con espacios más reducidos la $\mathrm{FC}$ del jugador

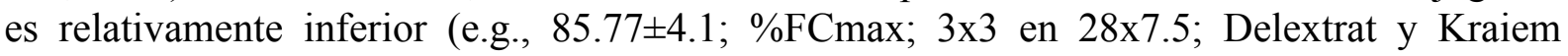
2013).

El estudio de Atli y col. (2013) y la comparación de estudios con espacios de juego diferentes, permiten sugerir que medidas de espacio superiores, llevaran a un incremento en los valores de FC del jugador y viceversa. Además, los datos sugieren que, a menor espacio, mayor es la intensidad del juego, y por lo contrario con un espacio de juego superior, menor será la intensidad del juego. Sin embargo, el número limitado de estudios que comparan directamente la intensidad de juego respecto a la medida del espacio reduce la consistencia de estas conclusiones.

Modificación de normas. Dos investigaciones han utilizado la modificación de las normas con el fin de estudiar variaciones en la FC (Conte y col., 2015b; Dehesa y col., 2015), además Conte y col. (2015b) también analizaron las variaciones en la intensidad del juego. Los estudios mostraron que el uso de la defensa individual (Dehesa y col., 2015) y/o la prohibición del bote (Conte y col., 2015b) promueve valores superiores de FC (e.g., 83.27 \%FCmax; 92.0 0.6 \%FCmax; Defensa individual; Prohibición del bote; Dehesa y col., 2015, Conte y col., 2015b). Contrariamente, la defensa zonal y la libre utilización del bote promueve valores inferiores de FC (e.g., $73.8 \%$ FCmax; 90.0 $\pm 0.5 \%$ FCmax; Defensa zonal; Libre utilización del bote; Dehesa y col., 2015; Conte y col., 2015b). No obstante, únicamente se hallaron diferencias significativas de variación de la $\mathrm{FC}$ según el tipo de defensa utilizada. En cuanto a la intensidad del juego, el estudio de Conte y col. (2015b) indica que cuando se prohíbe el bote se da una mayor intensidad del juego respecto a cuándo se permite una libre utilización del bote, encontrando diferencias significativas en cuanto a número de pases (e.g., 30.1 \pm 2.0 Total acciones pases; $14.9 \pm 1.3$ Total acciones pases; Prohibición del bote; Libre utilización del bote; Conte y col., 2015b).

Organización del tiempo. El intervalo de tiempo más utilizado fue de cuatro minutos (Tabla 1). Otros autores también utilizaron series de cinco minutos o dos minutos y medio (Klusseman y col., 2012), series de un minuto de trabajo y uno de descanso (Conte y col., 2016) u otras variantes de tres minutos, tres minutos y medio o cuatro minutos y cuarto (Delextrat y Martínez, 2014). Los tiempos de descansos fueron más variables y se establecieron descansos de uno, dos y tres minutos entre los diferentes estudios. En cuanto a los regímenes de trabajo, la mayoría de estudios se centraron en la utilización de regímenes continuos. El régimen continuo establece series continuas de trabajo en las que los jugadores están continuamente participando en la tarea, mientras que un régimen intermitente establece series de dos o más fracciones de trabajo con pequeños descansos entre ellos en las que los 
Sacot, A.; Escosa, J., y Latinjak, A. T. (2017). Propuesta Metodológica del Entrenamiento de la Resistencia en Baloncesto mediante la Modificación de Factores Formales y Estructurales del Juego. RICYDE. Revista internacional de ciencias del deporte, 50(13), 409-425. https://doi.org/10.5232/ricyde2017.05007

jugadores participan de forma alternada en la tarea, independientemente del tipo de régimen puede haber descansos entre series de la misma tarea.

Dos estudios compararon el efecto del tipo de régimen de trabajo sobre la FC y la intensidad del juego (Conte y col., 2016; Klusseman y col., 2012). Los resultados obtenidos por estas investigaciones indican que los regímenes de trabajo continuos, promueven una FCmax superior (e.g., 90.8 $\pm 2.7 \%$ FCmax; 86 \pm 4 \%FCmax; Conte y col., 2016; Klusseman y col., 2012 ) en comparación a los regímenes de trabajo intermitente (e.g., $86.5 \pm 3.7 \%$ FCmax; $83 \pm 3$ \%FCmax; Conte y col., 2016; Klusseman y col., 2012). Por otro lado, los regímenes de trabajo intermitentes, provocaron un aumento de la intensidad del juego debido a un mayor número de acciones de moderada y alta intensidad y mayores acciones técnicas (e.g., 21.0 0.3 Total acciones pases; 55 18 Total acciones técnicas; Conte y col., 2016; Klusseman y col., 2012) en comparación con los regímenes de trabajo continuos (e.g., 18.7 \pm 6.4 Total Pases; $51 \pm 17$ Total acciones técnicas; Conte y col., 2016; Klusseman y col., 2012).

En resumen, parámetros que precipitarían un aumento de la $\mathrm{FC}$ serian un reducido número de jugadores, desigualdades numéricas, mayor espacio, el trabajo con defensa individual, la prohibición del bote y regímenes de trabajo continuos. Además, parámetros que precipitarían un aumento de la intensidad del juego serian un número reducido de jugadores, desigualdades numéricas, menor espacio, el trabajo con defensa individual, la prohibición del bote y regímenes de trabajo intermitentes. Contrariamente, parámetros que precipitarían una disminución de la FC serian un número elevado de jugadores, con un menor espacio, trabajo con defensa zonal, la utilización del bote y regímenes de trabajo intermitentes. Asimismo, un número elevado de jugadores, mayor espacio, defensas zonales, la utilización del bote y regímenes de trabajo continuos precipitarían una disminución de la intensidad del jugo. Sin embargo, se cree pertinente sugerir que los diferentes parámetros no se dan en aislamiento los unos de los otros. 


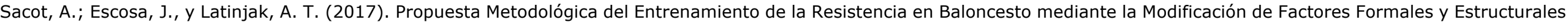
del Juego. RICYDE. Revista internacional de ciencias del deporte, 50(13), 409-425. https://doi.org/10.5232/ricyde2017.05007

Tabla 1. Resumen de los artículos incluidos en la revisión sistemática con datos sobre los parámetros de juego modificados

\begin{tabular}{|c|c|c|c|c|c|c|}
\hline Autor & Muestra & $\mathrm{N}^{0}$ jugadores & Medida del espacio & Ratio de trabajo & Acciones técnicas & Normas \\
\hline Atli y col., 2013 & $\begin{array}{l}12 \text { jugadoras } \\
(15 \text { años } \pm 0.5)\end{array}$ & $3 \times 3$ & $\begin{array}{l}\text { Medio campo y todo } \\
\text { el campo }\end{array}$ & $4 \times 4^{\prime}$ con $2{ }^{\prime}$ descanso & $\begin{array}{l}\text { Tiro, asistencia, rebotes, } \\
\text { recuperaciones, pase y } \\
\text { pérdidas }\end{array}$ & $\begin{array}{l}\text { Balones a punto para no perder } \\
\text { intensidad, animación verbal } \\
\text { entrenador }\end{array}$ \\
\hline Castagna y col., 2011 & $\begin{array}{l}14 \text { jugadores } \\
(18.9 \text { años } \pm 2.3)\end{array}$ & $5 \times 5,3 \times 3$ y $2 \times 2$ & Todo el campo & $2 \times 4^{\prime}$ & - & Defensa individual \\
\hline Conte y col., 2015b & $\begin{array}{l}23 \text { jugadores } \\
(15.5 \text { años } \pm 0.9)\end{array}$ & $4 \times 4$ & Todo el campo & $4^{\prime}$ con 2' de descanso & $\begin{array}{l}\text { Pases, tiros, intercepciones, } \\
\text { robos, perdidas y rebotes }\end{array}$ & $\begin{array}{l}\text { Defensa individual, limitación de } \\
\text { bote y sin limitación de bote }\end{array}$ \\
\hline Conte y col., 2016 & $\begin{array}{l}21 \text { jugadores Junior } \\
(15.4 \pm 0.9)\end{array}$ & $2 \times 2$ y $4 \times 4$ & Todo el campo & 3x4' y $3 \times 7$ '(1'treball + 1'descans $)$ & $\begin{array}{l}\text { Bote, robos, rebotes, } \\
\text { pérdidas, pase y tiro }\end{array}$ & $\begin{array}{l}\text { No tiros libres y balones } \\
\text { preparados para dar continuidad }\end{array}$ \\
\hline $\begin{array}{l}\text { Delextrat y Kraiem } \\
2013\end{array}$ & $\begin{array}{l}31 \text { jugadores Juniors } \\
\text { Bases }(16.6 \text { años } \pm 1.0) \\
\text { Aleros }(15.8 \text { años } \pm 0.9) \\
\text { Pívots }(16.1 \text { años } \pm 0.6)\end{array}$ & $2 \times 2$ y $3 \times 3$ & $\begin{array}{l}\text { Medio campo de todo } \\
\text { el campo }(28 \times 7.5)\end{array}$ & $\begin{array}{l}3 \times 44^{\prime} \\
1^{\prime} \text { descanso }\end{array}$ & - & $\begin{array}{l}\text { Inicio } 2 \text { fuera en el } 2 \times 2 \text { y } 2 \text { fuera } \\
\text { dentro en el } 3 \times 3 \text {, } \\
\text { Defensa individual } \\
\text { No tiros libres ni tiempos muertos }\end{array}$ \\
\hline $\begin{array}{l}\text { Delextrat y Martínez } \\
2014\end{array}$ & $\begin{array}{l}9 \text { jugadores Juniors }(16.3 \\
\text { años } \pm 0.8)\end{array}$ & $2 \times 2$ & $\begin{array}{l}\text { Medio campo de todo } \\
\text { el campo }(28 \times 7.5)\end{array}$ & $\begin{array}{l}2 \times\left(2 \times 3^{\prime} 45^{\prime \prime}\right), 2 \times\left(2 \times 4^{\prime} 15^{\prime \prime}\right), 2 \times\left(3 \times 3^{\prime}\right) \\
2 x\left(3 \times 3^{\prime} 30^{\prime \prime}\right), 2 \times\left(3 \times 4^{\prime}\right), 2 \times\left(3 \times 4^{\prime} 15^{\prime \prime}\right)\end{array}$ & - & $\begin{array}{l}\text { Defensa individual y no tiros } \\
\text { libres, marcador y animación } \\
\text { verbal entrenador }\end{array}$ \\
\hline Dehesa y col.,2015 & $\begin{array}{l}11 \text { jugadores } \\
\text { profesionales ( } 26.45 \text { años } \\
\pm 3.7)\end{array}$ & $\begin{array}{l}1 \times 1,2 \times 2,3 \times 3 \\
4 \times 4,5 \times 5, \text { SUP e } \\
\text { INF }\end{array}$ & Todo el campo & $\begin{array}{l}5.37 \pm 1.18(1 \times 1), 7.59 \pm 1.86(2 \times 2) \\
10.95 \pm 3.12(3 \times 3), 10.50 \pm 1.08(4 \times 4) \\
10.99 \pm 3.77(5 \times 5), 4.94 \pm 1.10(\text { SUP) } \\
9.34 \pm 2.77(\mathrm{INF})\end{array}$ & - & Defensa zonal y defensa individu: \\
\hline $\begin{array}{l}\text { Klusseman y col., } \\
2012\end{array}$ & $\begin{array}{l}8 \text { jugadores Juniors }(18.2 \\
\text { años } \pm 0.3 \text { ) } \\
8 \text { jugadores junior }(17.4 \\
\text { años } \pm 0.7)\end{array}$ & $2 \times 2$ y $4 \times 4$ & $\begin{array}{l}\text { Medio campo y todo } \\
\text { el campo }\end{array}$ & $2 \times 5^{\prime}$ y $4 \times 2{ }^{\prime} 30^{\prime \prime}$ & $\begin{array}{l}\text { Bote, pasada, tiro cerca de } \\
\text { canasta, tiro de media } \\
\text { distancia, tiro de } 3 \text { puntos, } \\
\text { rebote y bloqueo }\end{array}$ & $\begin{array}{l}\text { Juego libre, } 12 \text { segundos de } \\
\text { posesión, punto después de falta, } \\
\text { no tiros libres }\end{array}$ \\
\hline $\begin{array}{l}\text { Montgomery y col., } \\
2010\end{array}$ & $\begin{array}{l}11 \text { jugadores Junior }(19.1 \\
\text { años } \pm 2.1)\end{array}$ & $5 \times 5$ & Medio campo & - & - & - \\
\hline Reina y col., 2017 & $\begin{array}{l}14 \text { jugadoras senior } \\
\text { amateur }(21.7 \text { años } \pm 3.65)\end{array}$ & $\begin{array}{l}2 \times 2,3 \times 3,4 \times 4 \\
5 \times 5 \text { y } 5 \times 5 \text { (real) }\end{array}$ & Todo el campo & - & - & - \\
\hline $\begin{array}{l}\text { Torres-Ronda y col., } \\
2016\end{array}$ & $\begin{array}{l}14 \text { jugadores } \\
\text { profesionales ( } 25.5 \text { años } \\
\pm 4.7)\end{array}$ & $\begin{array}{l}\mathrm{Xx} 0,1 \mathrm{x} 1,2 \mathrm{xX} \\
2 \times 2,3 \times X, 3 \times 3 \\
4 \times X, 4 \times 4,5 \times 5 y \\
5 \times 5 \text { (real) }\end{array}$ & $\begin{array}{l}\text { Medio campo y todo } \\
\text { el campo }\end{array}$ & - & - & - \\
\hline Vaquera y col., 2017 & $\begin{array}{l}14 \text { jugadoras junior }(16.38 \\
\text { años } \pm 1.87)\end{array}$ & $\begin{array}{l}3 \times 3 \times 3 \times 3,4 \times 4 \times 4 \\
5 \times 5 \times 5 \text { y } 3 \times 3 \\
\text { continuo }\end{array}$ & Todo el campo & $\begin{array}{l}\text { Descansos de } 60,90,120 \text { y } 150 \\
\text { segundos variables entre semanas }\end{array}$ & - & - \\
\hline
\end{tabular}


Sacot, A.; Escosa, J., y Latinjak, A. T. (2017). Propuesta Metodológica del Entrenamiento de la Resistencia en Baloncesto mediante la Modificación de Factores Formales y Estructurales del Juego. RICYDE. Revista internacional de ciencias del deporte, 50(13), 409-425. https://doi.org/10.5232/ricyde2017.05007

Como conclusión, por un lado serían necesarios más estudios experimentales que comprobaran la interacción y los efectos sumatorios de los diferentes parámetros sobre la $\mathrm{FC}$ y la intensidad de jugo y por otro lado, se cree necesario integrar los diferentes resultados obtenidos sobre los efectos de los parámetros en la FC y la intensidad de juego para la creación de una propuesta metodológica que sea de utilidad para el entrenamiento integrado de la resistencia junto con elementos técnico-tácticos en baloncesto.

\section{Propuesta metodológica}

La propuesta metodológica para el trabajo de la resistencia específica en baloncesto mediante los juegos en espacios reducidos se fundamenta en los diferentes resultados de la presente revisión y en la idea de trabajo de la resistencia en los deportes de equipo de Seirul·lo (1987) y Solé (2002). Desde éste punto de vista, el entrenamiento no sólo tendría una orientación específica hacia una estructura, sino que las diferentes estructuras se verían implicadas aunque con ciertos focos en unas u otras en función del objetivo de las tareas y del sistema de juego del equipo (Seirul·lo, 1987). Por ejemplo, una situación de $3 \times 3$ continuado a toda la pista, puede estar focalizado en la organización de salida del contraataque, con la finalidad de incidir sobre la estructura cognitiva. Aun así, es evidente que éste ejercicio requiere una participación de la estructura condicional (i.e., carga fisiológica sobre el organismo), coordinativa (i.e., movimientos y acciones concretas para realizar la salida de contraataque según el modelo de juego y la posición en el campo), socio-afectiva (i.e., interacciones e interrelaciones con sus compañeros constantes), emotivo-volitiva (i.e., gestión a nivel emocional) y creativo-expresiva (i.e., adaptación a las acciones de los defensas hecho que puede promover la utilización de nuevos movimientos). De este modo, un mismo ejercicio podría tener distintas orientaciones u objetivos en función del foco durante la tarea.

Asimismo, desde esta percepción holística, no se entendería la participación de forma aislada de un sistema energético u otro, sino que en todas las tareas existe una participación conjunta de los dos sistemas energéticos de obtención de energía durante el ejercicio: aeróbico y anaeróbico. Sin embargo, se podría aumentar la participación de uno u otro en función del objetivo de las tareas del entrenamiento y del sistema de juego del equipo. Siguiendo con ésta idea, desde el punto de vista de los autores, el equipo técnico, de forma integrada, debería disponer de herramientas para diseñar juegos en espacios reducidos con el fin de trabajar la resistencia, adaptados a la idea de juego del equipo y las condiciones específicas de juego de la competición más próxima.

Así pues, tal y como se puede observar en la Figura 2, el rectángulo superior representa el organismo fraccionado con los dos sistemas, aeróbico y anaeróbico, divididos en partes desiguales según su grado de participación. El lado izquierdo representa una participación mayoritaria del sistema anaeróbico, mientras que el lado derecho representa una mayor

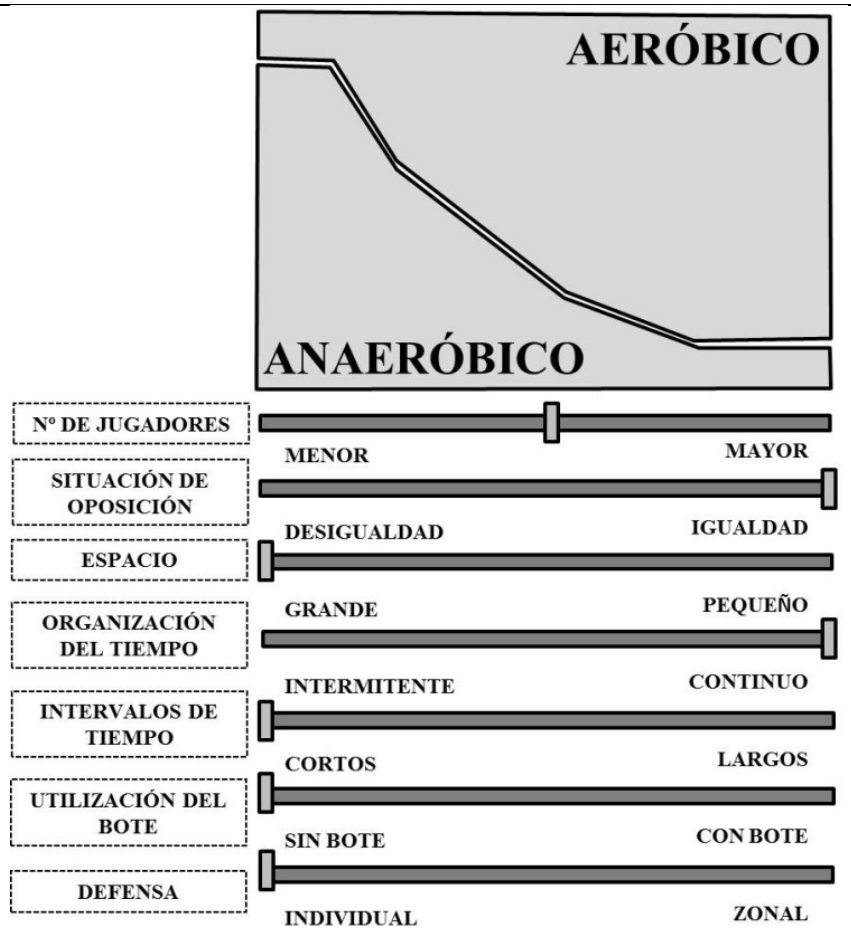

Figura 2. Ecualizador con los diferentes parámetros de juego y su sistema energético predominante 
Sacot, A.; Escosa, J., y Latinjak, A. T. (2017). Propuesta Metodológica del Entrenamiento de la Resistencia en Baloncesto mediante la Modificación de Factores Formales y Estructurales del Juego. RICYDE. Revista internacional de ciencias del deporte, 50(13), 409-425. https://doi.org/10.5232/ricyde2017.05007

participación del sistema aeróbico. De este modo, la parte central representa una participación proporcionalmente similar de los dos sistemas energéticos. Conforme aumenta la participación de un sistema energético el otro disminuye su participación y viceversa, hecho que representa la interacción constante del sistema aeróbico y anaeróbico en la obtención de energía durante el trabajo de la resistencia.

A partir de ahí, se pueden observar los distintos parámetros del juego extraídos de la revisión, distribuidos según el grado de participación del sistema aeróbico o anaeróbico en base a los resultados de los estudios analizados. En la parte correspondiente a la mayor participación del sistema anaeróbico respecto al aeróbico se pueden observar aquellas características vinculadas a un aumento de la FC y/o la intensidad del juego: un menor número de jugadores, desigualdades numéricas, espacios de juego grandes, organizaciones del tiempo intermitentes, tiempos de trabajo cortos, no utilización del bote y utilización de defensa individual. En la parte referente a la mayor participación del sistema aeróbico respecto al anaeróbico encontramos aquellas características vinculadas a menor FC y/o la intensidad del juego: un mayor número de jugadores, igualdades numéricas, espacios de juego pequeños, organizaciones del tiempo continuas, tiempos de trabajo largos, libre utilización del bote y utilización de defensa zonal. En cuanto a la organización del tiempo sería necesario puntualizar que los estudios analizados utilizaron ejercicios claramente continuos 0 intermitentes con la finalidad de aislar bien cada tarea. Sin embargo, en el entrenamiento es posible encontrar ejercicios continuos en cuanto a tiempo, pero con participación intermitente del jugador como por ejemplo un $2 \times 2$ continuado a toda la pista. En este caso, el panel se colocaría en la parte central del ecualizador puesto que hasta donde llega el conocimiento de los autores no se puede establecer con seguridad si existe una participación clara de un sistema sobre el otro. Acerca de la situación de oposición, los diferentes estudios también han utilizado tareas con una oposición permanentemente igual o desigual. No obstante, durante el entrenamiento se pueden encontrar tareas las cuales partan de una situación de igualdad pero que durante su transcurso se produzcan oposiciones desiguales de forma momentánea, como por ejemplo el mismo $2 \times 2$ continuado a toda la pista. En este caso, también se colocaría el panel en la parte central del ecualizador puesto que hasta donde llega el conocimiento de los autores no se puede establecer con seguridad si existe una participación clara de un sistema sobre el otro.

Llegados a este punto, la herramienta parte de la idea de un ecualizador de sonido, donde existen distintos parámetros, en este caso de la situación de juego, que pueden ser ajustados y distribuidos con la finalidad que el producto final sea lo más ajustado a la realidad que se precisa resolver. La herramienta podría tener dos distintos usos: el control del entrenamiento en tareas previamente planificadas o la creación de tareas. En primera instancia, ésta propuesta se podría utilizar como herramienta de control del entrenamiento con la finalidad de conocer a posteriori el tipo de resistencia predominante durante el entrenamiento y así poder ajustar la planificación según los requerimientos de la competición. Además, la propuesta también puede ser utilizada con un propósito de creación de tareas. Sírvase de ejemplo un equipo técnico tiene como objetivo principal de la tarea el trabajo de la resistencia anaeróbica integrado a un trabajo del bloqueo directo en situaciones de $2 \times 2$ contra defensas individuales. En este caso, partiendo de la base de una situación de 2x2, el equipo técnico tendría que discutir la gestión de los distintos parámetros para conseguir la orientación hacia el sistema anaeróbico. 
Sacot, A.; Escosa, J., y Latinjak, A. T. (2017). Propuesta Metodológica del Entrenamiento de la Resistencia en Baloncesto mediante la Modificación de Factores Formales y Estructurales del Juego. RICYDE. Revista internacional de ciencias del deporte, 50(13), 409-425. https://doi.org/10.5232/ricyde2017.05007

En la creación de tareas mediante esta herramienta, se pueden observar tres tipos de orientaciones del trabajo: orientación anaeróbica, con una predominancia del sistema anaeróbico respecto al aeróbico; orientación aeróbica, con una predominancia del sistema aeróbico respecto al anaeróbico; y una orientación equilibrada, con una implicación más equitativa del sistema anaeróbico y aeróbico. En primer lugar, si el objetivo de la tarea fuese en el trabajo de la resistencia anaeróbica, sería imprescindible que la mayoría de los paneles se encontrasen desplazados en la parte izquierda del ecualizador. Sírvase de ejemplo un ejercicio comúnmente utilizado por entrenadores como podría una situación de 1x1 a toda la pista. Se trata de una situación con igualdad numérica, donde se utiliza todo el campo, con una organización intermitente con 6 series de entre 10 y 20 segundos de trabajo, con bote y mediante defensa individual (Figura 3a). En segundo lugar, si el foco de la tarea fuera en el trabajo de la resistencia aeróbica, sería imprescindible que la mayoría de los paneles fuesen situados en la parte derecha del ecualizador. Sírvase de ejemplo un ejercicio muy utilizado para el trabajo táctico colectivo como es el 5x5 a medio campo. En este caso, se utiliza una situación de $5 \times 5$, en igualdad numérica, con medio campo de juego, con una organización continua con una serie de 6 minutos de trabajo, con bote y con defensa individual (Figura 3b). Finalmente, si el objetivo de la tarea se focalizara en un trabajo equilibrado, los paneles deberían estar situados en la zona central o bien situados entre ambos lados del ecualizador, con un mayor equilibrio entre los dos lados. Póngase de ejemplo una situación muy empleada por los entrenadores como podría ser un $3 \times 3$ continuado. En éste caso, se utiliza una situación de $3 \times 3$ (situación de oposición intermedia que supone situar el panel en la zona central); con igualdades pero con momentos de superioridad numérica (se situaría el panel situación de oposición en el centro puesto que no se trataría de una situación siempre igual o desigual), a todo el campo, con un trabajo continuo de 6 minutos, pero con participación fraccionada del jugador de 25 segundos de trabajo con 25 segundos de descanso (se situaría el panel organización del tiempo en la zona central puesto que se trata de un trabajo continuo e intermitente de forma simultánea), con bote $y$ una defensa individual (Figura 3c). 


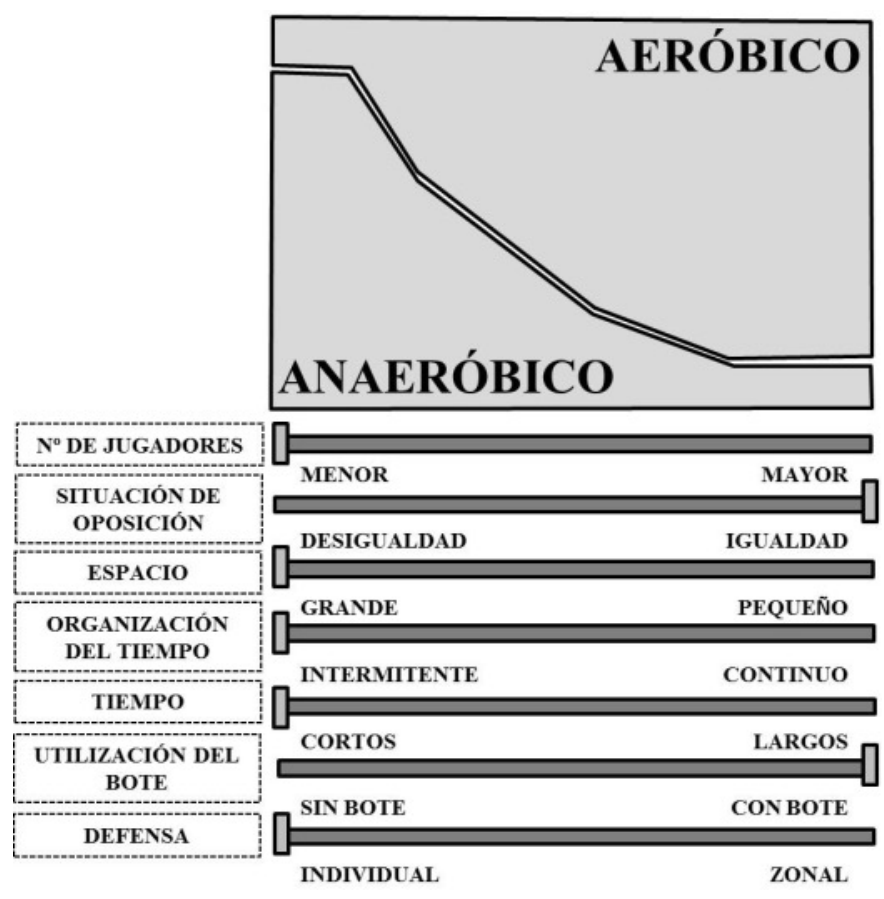

Figura 3a. Situación $1 \mathrm{x} 1$ a toda la pista

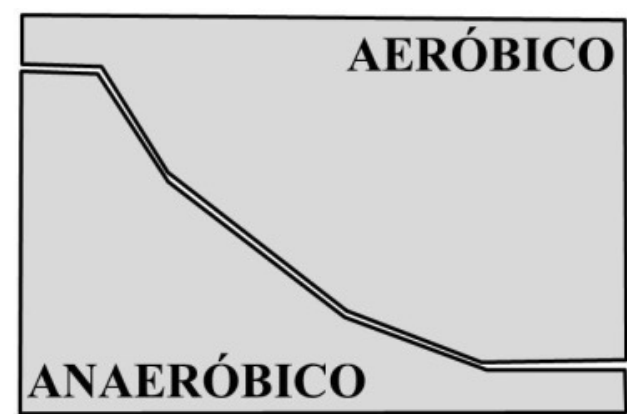

\begin{tabular}{|c|c|c|}
\hline $\mathrm{N}^{\circ}$ DE JUGADORES & \multirow{2}{*}{ MENOR } & \multirow{2}{*}{ MAYOR } \\
\hline SITUACIÓN DE & & \\
\hline OPOSICION & \multirow{2}{*}{ DESIGUALDAD } & \multirow{2}{*}{ IGUALDAD } \\
\hline ESPACIO & & \\
\hline ORGANIZACIÓN & GRANDE & PEQUEÑO \\
\hline DEL TIEMPO & \multirow{2}{*}{ INTERMITENTE } & \multirow{2}{*}{ CONTINUO } \\
\hline TIEMPO & & \\
\hline UTILIZACIÓN DEL & CORTOS & LARGOS \\
\hline BOTE & \multirow{2}{*}{ SIN BOTE } & \multirow{2}{*}{ CONBOTE } \\
\hline DEFENSA & & \\
\hline
\end{tabular}

Figura 3 b. Situación $5 \times 5$ a medio campo

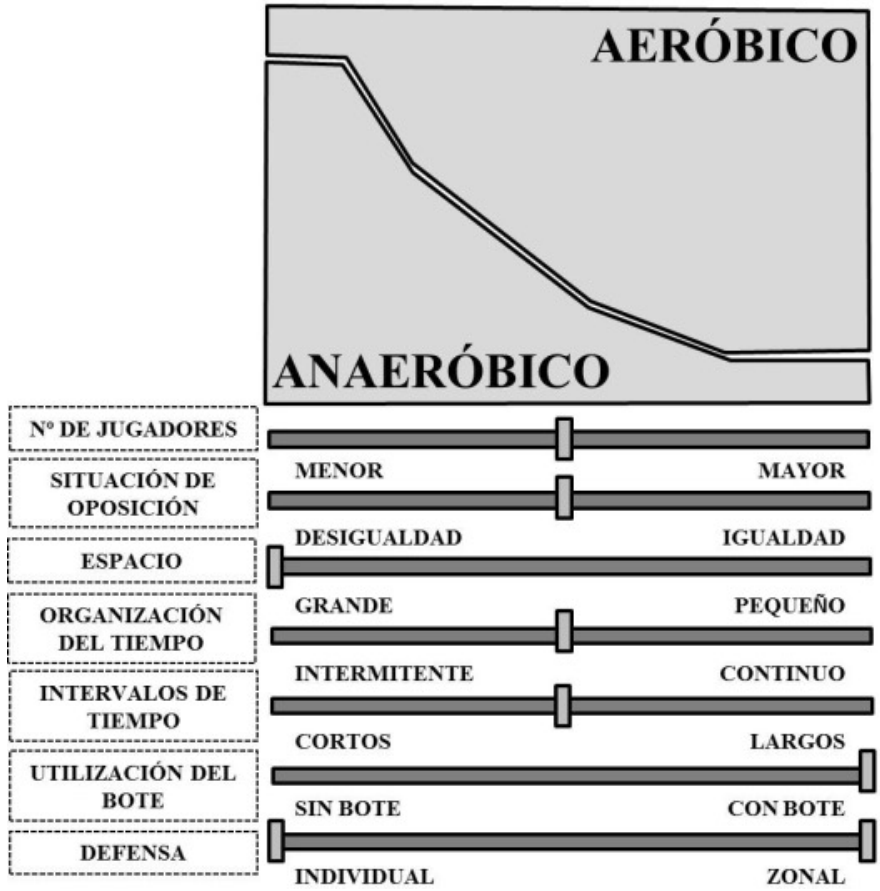

Figura $3 c$. Situación $3 \times 3$ continuado 
Sacot, A.; Escosa, J., y Latinjak, A. T. (2017). Propuesta Metodológica del Entrenamiento de la Resistencia en Baloncesto mediante la Modificación de Factores Formales y Estructurales del Juego. RICYDE. Revista internacional de ciencias del deporte, 50(13), 409-425. https://doi.org/10.5232/ricyde2017.05007

\section{Limitaciones y líneas de estudio futuras}

En primer lugar, se encontraron limitaciones en relación al área de estudio. Hasta dónde alcanza el conocimiento de los autores, existen pocos estudios que hayan analizado el efecto sobre la FC y/o la intensidad de juego de distintas situaciones de juego en espacios reducidos en baloncesto. Además, estos estudios han analizado pocos parámetros del juego y de forma simplificada. Este hecho limita la propuesta metodológica puesto que se requiere un conocimiento exhaustivo del efecto de éstos parámetros sobre el organismo con el fin de ajustar aún más el trabajo de la resistencia a la realidad competitiva.

En segundo lugar, los diferentes estudios no han analizado las distintas interacciones que existen entre los parámetros del juego. Éste hecho condiciona la propuesta metodológica ya que por ejemplo, una situación de $5 \times 5$ implicaría una mayor participación del sistema aeróbico respecto al anaeróbico y jugar con un espació grande, implicaría una mayor participación del sistema anaeróbico respecto al aeróbico. Aun así, no se puede saber con certeza si el hecho de jugar este $5 \times 5$ a tota la pista implicara más la utilización de un sistema u otro porque no sabemos cómo interaccionan éstas variables entre sí. Así pues, nuestra propuesta se basa en los efectos simples de los diferentes parámetros y su validación depende, entre otras, de estudios que analizan la interacción entre distintos parámetros.

La propuesta metodológica se ha basado en los cambios en la FC y la intensidad de juego en función de distintos parámetros de juego implicados durante los juegos en espacio reducido. Aun así, diferentes autores (Nazaraki y col., 2008; Fox y col., 2017) coinciden en que la FC de forma aislada no sería una herramienta fiable para el control de la carga interna en baloncesto debido a que las características del deporte producen una alteración y una sobreestimación de la FC. Por este motivo, éste hecho supone una limitación importante ya que como proponen Fox y col. (2017), para aumentar la validez de la FC debería ser contrastada mediante otros métodos de control. En éste caso, se ha contrastado con elementos de carga externa como son las acciones técnicas como variable de intensidad. Sin embargo, debería ser comparada con otros métodos para poder sacar conclusiones más certeras y así poder aproximar la propuesta a la realidad del baloncesto.

Finalmente, la propuesta metodológica está centrada en los parámetros del juego, en ningún caso sobre el jugador. Éste es un factor importante puesto que sabemos cómo afectan estos parámetros del juego en la FC de los grupos de jugadores de los distintos estudios, pero no podemos presuponer que todos los parámetros del juego van a tener siempre el mismo efecto $\mathrm{y}$ en todos los jugadores por igual. Consecuentemente, los entrenadores no deben perder de vista la respuesta de cada jugador a los ejercicios que se proponen y se llevan a cabo.

Resumiendo, de cara a futuros estudios sugerimos que se pueden utilizar otros parámetros para ver su efecto sobre la FC y/o la intensidad del juego, además, se podrían combinar distintos parámetros en un mismo estudio y analizar el grado de interacción entre los distintos parámetros del juego. También se podrían emplear otras variables dependientes de carga interna o externa para obtener más información y así poder ajustar más la propuesta metodológica para el trabajo de la resistencia. 
Sacot, A.; Escosa, J., y Latinjak, A. T. (2017). Propuesta Metodológica del Entrenamiento de la Resistencia en Baloncesto mediante la Modificación de Factores Formales y Estructurales del Juego. RICYDE. Revista internacional de ciencias del deporte, 50(13), 409-425. https://doi.org/10.5232/ricyde2017.05007

\section{Conclusiones}

En este estudio se ha llevado a cabo una revisión sistemática sobre los distintos parámetros de los juegos en espacio reducido y sus efectos sobre la FC y la intensidad de juego. La búsqueda ha resultado un número relativamente moderado de artículos teniendo en cuenta la gran variedad de parámetros que se han estudiado en ellos. Como consecuencia, se encuentra poca replicación de los resultados hallados por los diferentes estudios, hecho que limita la confianza en las conclusiones que se pueden obtener. Sin embargo, teniendo en cuenta ésta limitación hemos podido elaborar una propuesta metodológica, en forma de ecualizador, pensada para que equipos técnicos puedan controlar y/o crear tareas de entrenamiento con un trabajo integrado de la resistencia y el trabajo técnico-táctico. A día de hoy, podemos afirmar que parámetros como el tamaño del espacio, condiciones en relación al número de jugadores, parámetros temporales y normas de juego, pueden ser claves en el momento de integrar el trabajo de la resistencia específica en baloncesto y los juegos en espacio reducido.

\section{Referencias}

Atli, H.; Köklü, Y.; Alemdaroglu, U., y Koçak, F. Ü. (2013). A comparison of heart rate response and frequencies of technical actions between half-court and full-court 3-aside games in high school female basketball players. The Journal of Strength and Conditioning Research, 27(2), 352-356.

https://doi.org/10.1519/JSC.0b013e3182542674

Balagué, N.; Torrents, C.; Pol, R., y Seirul·lo, F. (2014). Entrenamiento integrado. Principios dinámicos y aplicaciones. Apuntes. Educación física y deporte, 2(116), 6068. https://doi.org/10.5672/apunts.2014-0983.es.(2014/2).116.06

Ben Abdelkrim, N.; Castagna, C.; El Fazaa, S., y El Ati, J. (2010a). The effect of players' standard and tactical strategy on game demands in men's basketball. The Journal of Strength and Conditioning Research, 24(10), 2652-2662. https://doi.org/10.1519/JSC.0b013e3181e2e0a3

Ben Abdelkrim, N.; Castagna, C.; Jabri, Y.; Battikh, T.; El Fazaa, S., y El Ati, J. (2010b). Activity profile and physiological requirements of junior elite basketball players in relation to aerobic-anaerobic fitness. The Journal of Strength and Conditioning Research, 24(9), 2330-2342.

https://doi.org/10.1519/JSC.0b013e3181e381c1

Ben Abdelkrim, N.; El Fazaa, S., y El Ati, J. (2007). Time-motion analysis and physiological data of elite under-19-year-old basketball players during competition. British Journal of Sports Medicine, 41(2), 69-75.

https://doi.org/10.1136/bjsm.2006.032318

Castagna, C.; Impellizzeri, F. M.; Chaouachi, A.; Ben Abdelkrim, N., y Manzi, V. (2011). Physiological responses to ball-drills in regional level male basketball players. Journal of Sports Sciences, 29(12), 1329-1336.

https://doi.org/10.1080/02640414.2011.597418

Conte, D.; Favero, T. G.; Lupo, C.; Francioni, F. M.; Capranica, L., y Tessitore, A. (2015a). Time-motion analysis of Italian elite women's basketball games: Individual and team analysis. The Journal of Strength and Conditioning Research, 29(1), 144150.

https://doi.org/10.1519/JSC.0000000000000633

Conte, D.; Favero, T. G.; Niederhausen, M.; Capranica, L., y Tessitore, A. (2015b). Physiological and technical demands of no dribble game drill in young basketball players. The Journal of Strength and Conditioning Research, 29(12), 3375-3379.

https://doi.org/10.1519/JSC.0000000000000997 
Sacot, A.; Escosa, J., y Latinjak, A. T. (2017). Propuesta Metodológica del Entrenamiento de la Resistencia en Baloncesto mediante la Modificación de Factores Formales y Estructurales del Juego. RICYDE. Revista internacional de ciencias del deporte, 50(13), 409-425. https://doi.org/10.5232/ricyde2017.05007

Conte, D.; Favero, T. G.; Niederhausen, M.; Capranica, L., y Tessitore, A. (2016). Effect of different number of players and training regimes on physiological and technical demands of ball-drills in basketball. Journal of Sports Sciences, 34(8), 780-786. https://doi.org/10.1080/02640414.2015.1069384

Davids, K.; Araújo, D.; Correia, V., y Vilar, L. (2013). How small-sided and conditioned games enhance acquisition of moviment and decision-making skills. Exercise and Sport Sciences Reviews, 41(3), 154-161. https://doi.org/10.1097/JES.0b013e318292f3ec

Dehesa, R.; Vaquera, A.; García-Tormo, J.V., y Bayón, P. (2015). Heart rate analysis of high level basketball players during training sessions. Revista de Psicología del Deporte, 24(1), 17-19.

Delextrat, A.; Badiella, A.; Saavedra, V.; Matthew, D.; Schelling, X., y Torres-Ronda, L. (2015). Match activity demands of elite Spanish female basketball players by playing position. International Journal of Performance Analysis in Sport, 15(2), 687-703.

Delextrat, A.; Baliqi, F., y Clarke, N. (2013). Repeated sprint ability and stride kinematics are altered following an oficial match in national-level basketball players. The Journal of Sports Medicine and Physical Fitness, 52(2), 112-118.

Delextrat, A., y Cohen, D. (2009). Strength, power, speed, and agility of women basketball players according to playing position. The Journal of Strength and Conditioning Research, 23(7), 1974-1981.

https://doi.org/10.1519/JSC.0b013e3181b86a7e

Delextrat, A., y Kraiem, S. (2013). Heart-rate responses by playing position during ball drills in basketball. International Journal of Sports Physiology and Performance, 8(4), 410-418.

https://doi.org/10.1519/JSC.0b013e3181b86a7e

Delextrat, A., y Martinez, A. (2014). Small-sided games training improves aerobic capacity and technical skills in basketball players. International Journal of Sports Medicine, 35(5), 385-391.

Fox, J. L., Scanlan, A. T., y Stanton, R. (2017). A review of player monitoring approaches in basketball: Current trends and future directions. The Journal of Strength and Conditioning Research, 31(7), 2021-2029 https://doi.org/10.1519/JSC.0000000000001964

Halouani, J.; Chtourou, H.; Gabbett, T.; Chaouachi, A., y Chamari, K. (2014). Smallsided games in team sports training: A brief review. The Journal of Strength and Conditioning Research, 28(12), 3594-3618.

https://doi.org/10.1519/JSC.0000000000000564

Kelso, J. S., y Tuller, B. (1984). A dynamical basis for action systems. In M.S. Gazzaniga (Ed.), Handbook of Cognitive Neuroscience (pp. 321-356). Springer US. https://doi.org/10.1007/978-1-4899-2177-2_16

Klusemann, M. J.; Pyne, D. B.; Foster, C., y Drinkwater, E. J. (2012). Optimising technical skills and physical loading in small-sided basketball games. Journal of sports Sciences, 30(14), 1463-1471. https://doi.org/10.1080/02640414.2012.712714

Klusemann, M. J.; Pyne, D. B.; Hopkins, W. G., y Drinkwater, E. J. (2013). Activity profiles and demands of seasonal and tournament basketball competition. International Journal of Sports Physiology and Performance, 8(6), 623-629. https://doi.org/10.1123/ijspp.8.6.623

Martínez-Fernández, S., García, J., \& Ibáñez Godoy, S. J. (2015). Incidence of type of game mode in player participation in minibasket. Revista de Psicología del Deporte, 24(3), 0065-68. 
Sacot, A.; Escosa, J., y Latinjak, A. T. (2017). Propuesta Metodológica del Entrenamiento de la Resistencia en Baloncesto mediante la Modificación de Factores Formales y Estructurales del Juego. RICYDE. Revista internacional de ciencias del deporte, 50(13), 409-425. https://doi.org/10.5232/ricyde2017.05007

Matthew, D., y Delextrat, A. (2009). Heart rate, blood lactate concentration, and timemotion analysis of female basketball players during competition. Journal of Sports Sciences, 27(8), 813-821.

https://doi.org/10.1080/02640410902926420

Montgomery, P. G.; Pyne, D. B., y Minahan, C. L. (2010). The physical and physiological demands of basketball training and competition. International Journal of Sports Physiology and Performance, 5(1), 75-86.

https://doi.org/10.1123/ijspp.5.1.75

Narazaki, K.; Berg, K.; Stergiou, N., y Chen, B. (2009). Physiological demands of competitive basketball. Scandinavian Journal of Medicine Science in Sports, 19(3), 425-432.

https://doi.org/10.1111/j.1600-0838.2008.00789.x

Nikolaidis, P.; Calleja-González, J., y Padulo, J. (2014). The effect of age on positional differences in anthropometry, bodycomposition, physique and anaerobic power of elite basketball players. Sport Sciences for Health, 10(3), 225-233.

https://doi.org/10.1111/j.1600-0838.2008.00789.x

Reina Román, M., Mancha Triguero, D., Feu Molina, S., y Ibáñez Godoy, S. J. (2017). ¿ Se entrena como se compite? Análisis de la carga en baloncesto femenino. Revista de Psicología del Deporte, 26(3), 0009-13.

Schelling, X., y Torres-Ronda, L. (2013). Conditioning for basketball: Quality and quantity of training. The Journal of Strength and Conditioning Research, 35(6), 89-94. https://doi.org/10.1519/SSC.0000000000000018

Seirul·lo, F. (1987). Opción de planificación en deportes de largo periodo de competiciones. Revista Entrenamiento Deportivo, 1(3), 53-62

Solé, J. (2002). Entrenamiento de la Resistencia en los deportes de equipo. Apuntes del Máster de alto rendimiento en deportes de equipo. Byomedic System: Barcelona

Torres-Ronda, L.; Ric, A.; Llabres-Torres, I.; de las Heras, B., y Schelling, X. (2016). Position-dependent cardiovascular response and time-motion analysis during training drills and friendly matches in elite male basketball players. The Journal of Strength and Conditioning Research, 30(1), 60-70. https://doi.org/10.1519/JSC.0000000000001043

Vaquera, A.; Suárez, D.; Vidania de Luis, L., y Calleja, J. (2017). Nueva aproximación a los juegos reducidos en baloncesto en función del número de jugadores, la percepción subjetiva del esfuerzo y la recuperación. Revista de Psicología del Deporte, 26(3), 0015-21. 